\title{
ALOJAMENTO CONJUNTO: ESPAÇO CONCRETO DE POSSIBILIDADES E O PAPEL DA EQUIPE MULTIPROFISSIONAL
}

\author{
Kelly Karine Pasqual ${ }^{1}$, Luzmarina Aparecida Doretto Braccialli², Mirela Volponi ${ }^{3}$
}

\begin{abstract}
RESUMO: Objetivou-se conhecer as possibilidades e os limites existentes no Sistema de Alojamento Conjunto (SAC) e analisar o papel da equipe multiprofissional inserida nesse local. Trata-se de uma revisão bibliográfica, a partir de 33 artigos publicados na base de dados Lilacs, entre 1990 e 2007. Constatou-se que o SAC apresenta inúmeras possibilidades, como a formação precoce de vínculo mãe-filho, incentivo à prática do aleitamento materno exclusivo, aproximação com a equipe multiprofissional, e limites relacionados a estrutura física inadequada, capacitação deficiente dos profissionais envolvidos, condições físicas e/ou emocionais inadequadas para o cuidado com o recém-nascido por parte da puérpera. Os profissionais necessitam trabalhar com criatividade e atualização de conhecimentos que estão diretamente ligadas a ações educativas efetivas. Sugere-se a atualização e o envolvimento dos profissionais com o SAC, com a efetivação de estratégias de educação permanente, em busca da melhoria da qualidade da assistência.
\end{abstract}

PALAVRAS-CHAVE: Alojamento conjunto; Aleitamento materno; Enfermagem; Equipe de assistência ao paciente.

\section{ROOMING-IN: AFACTUAL SETTING OF POSSIBILITIES AND THE ROLE OF MULTIDISCIPLINARYTEAMS}

\begin{abstract}
The aim of this study was to know the possibilities and boundaries that are present in the Rooming-In System (RIS) and to analyze the multi professional team which works in this setting. It is a bibliographic revision, from 33 papers published at Lilacs database between 1990 and 2007. We've found that the RIS presents multiple possibilities, such as the premature link mom-baby statement, the incentive to the exclusive breastfeeding practice, the approximation with the multi professional team. Limits are related to the inadequate physical structure, deficient training of the involved professionals, and inadequate physical or emotional conditions of the mother on caring for the newborn. Professionals need to work with creativity and to update the knowledge related to effective educational strategies. We suggest that this knowledge updating and professional involvement with the RIS should occur by the deployment of permanent education strategies, in order to enhance the quality of care.
\end{abstract}

KEYWORDS: Rooming-In services; Breastfeeding; Nursing; Patient care team.

\section{ALOJAMIENTO CONJUNTO: ESPACIO CONCRETO DE POSIBILIDADES Y EL PAPEL DELEQUIPO MULTIPROFESIONAL}

RESUMEN: Se objetivó conocer las posibilidades y los limites existentes en el Sistema de Alojamiento Conjunto (SAC) y analizar el papel del equipo multiprofesional inserido en ese local. Se trata de una revisión bibliográfica, a partir de 33 artículos publicados en la base de datos Lilacs entre 1990 y 2007. Se constató que el SAC presenta inúmeras posibilidades, como la formación precoz de vínculo madre-hijo, incentivo a la práctica del amamantamiento materno exclusivo, aproximación con el equipo multiprofesional, y limites relacionados a estructura física inadecuada, capacitación deficiente de los profesionales envueltos, condiciones físicas y/o emocionales inadecuadas para el cuidado con el recién-nacido por parte de la puérpera. Los profesionales necesitan trabajar con creatividad y actualización de conocimiento que están directamente ligadas a acciones educativas efectivas. Se sugiere actualización y envolvimiento de los profesionales con el SAC, con la efectuación de estrategias de educación permanente, en busca de la mejoría de la calidad de la asistencia.

PALABRAS CLAVE: Alojamiento conjunto; Amamantamiento materno; Enfermería; Grupo de atención al paciente.

\footnotetext{
${ }^{1}$ Acadêmica do Curso de Graudação em Enfermagem da Faculdade de Medicina de Marília-FAMEMA.

${ }^{2}$ Enfermeira. Doutora em Ciências. Docente da FAMEMA.

${ }^{3}$ Enfermeira. Residente do Programa de Residência Multiprofissional em Saúde da Família da FAMEMA.
}

Autor correspondente:

Mirela Volponi

Faculdade de Medicina de Marília

Av. Doutor Hélio Gomes Golveic, 220 - 17514-471 - Marília-SP, Brasil

Recebido: 30/07/09

E-mail: mirela.volponi@hotmail.com

Aprovado: 24/02/10

Cogitare Enferm. 2010 Abr/Jun; 15(2):334-9 


\section{INTRODUÇÃO}

No século $\mathrm{XV}$, a maioria das mulheres dava à luz em suas casas, assistidas por parteiras e acompanhadas pela família. Assim, o binômio mãe-filho se formava de uma maneira mais natural e fisiológica, possibilitando à mulher o cuidar do seu filho desde 0 nascimento. A partir dos séculos XVI e XVII, os hospitais tornaram-se instituições com credibilidade e os médicos atingiram o posto de comando devido ao embasamento na racionalidade científica moderna. Porém, só a partir do século XX os partos passaram a ocorrer, predominantemente, no ambiente hospitalar, agregando normas e rotinas. Nesse período, mãe e filho eram separados, as mulheres ficavam em unidades de puerpério e os bebês eram isolados em berçários ${ }^{(1)}$. Já nos anos 40, com o surgimento de estudos acerca da saúde emocional do ser humano - liderados por Edith Jackson e Grover Powers, ambos psiquiatras e docentes de pediatria - foi constatado que essa separação do binômio, por muitos considerada de grande valia na prevenção de contaminação, gerava receio e insegurança nas mães ${ }^{(1)}$.

Estudos realizados e movimentos sociais de mulheres que exigiam a permanência dos bebês com as mães após o parto levaram ao desenvolvimento de um projeto de alojamento conjunto, denominado Rooming-in Unit, com o intuito de humanizar o nascimento e promover o aleitamento materno.

O Alojamento Conjunto é um sistema hospitalar em que o recém-nascido sadio, logo após o nascimento, permanece ao lado da mãe 24 horas por dia, num mesmo ambiente, até a alta hospitalar. Este sistema permite à equipe multiprofissional realizar o cuidado direto, o controle do ambiente e a articulação com outros setores, possibilitando a prevenção de infecções e contribuindo para a saúde do binômio mãe-filho. O recém-nascido a termo necessita ter vitalidade e capacidade sucção, além de controle térmico adequado.

No Brasil, a primeira experiência de Alojamento Conjunto ocorreu em 1971, em Brasília. Em meados dos anos 80, a Organização Mundial de Saúde, o Ministério da Saúde e o UNICEF determinaram a implementação do Alojamento Conjunto, baseados na elevada taxa de desmame precoce no país. Em 26 de agosto de 1993, a Portaria n. 1016 foi aprovada, com a intenção de implementar as normas básicas do Sistema de Alojamento Conjunto (SAC), considerando a necessidade de incentivar o aleitamento e o relacionamento mãe-filho, diminuir o risco de infecção hospitalar e evitar as complicações maternas e do recém-nascido(2). O SAC foi implantado no Hospital das Clínicas de Marília - Unidade Materno-Infantil, na década de 70. No final de 2002, a instituição recebeu do Ministério da Saúde o título de "Hospital Amigo da Criança", após avaliação das ações desenvolvidas no cumprimento dos passos para o sucesso do aleitamento materno.

Com vistas a aprimorar o conhecimento das estudantes do Curso de Enfermagem acerca do SAC e das ações da equipe multiprofissional desenvolvidas nesse sistema, propusemo-nos a elaborar o presente trabalho, o qual tem como objetivo conhecer as possibilidades e os limites do SAC, bem como analisar o papel da equipe multiprofissional inserida nesse local.

\section{METODOLOGIA}

O presente estudo tem origem em Trabalho de Conclusão de Curso (TCC) realizado durante a $3^{\mathrm{a}}$ e $4^{\mathrm{a}}$ séries do Curso de Enfermagem da Faculdade de Medicina de Marília (Famema), nos anos de 2007 e 2008. Constitui-se de uma revisão bibliográfica, entendida como o levantamento da bibliografia de relevante interesse referente ao assunto que se deseja estudar, evitando o desperdício de tempo com temas já abordados $^{(3)}$. A revisão bibliográfica, também reconhecida como de fontes secundárias, retrata o levantamento de toda bibliografia já publicada, sendo esta em forma de livros, revistas, publicações avulsas e imprensa escrita. Seu propósito é colocar o pesquisador em contato direito com o que foi escrito sobre determinado assunto. A pesquisa bibliográfica pode ser considerada também como o primeiro passo de toda a pesquisa científica $^{(4)}$.

Foram utilizados 33 artigos publicados entre 1990 e 2007 na base de dados Lilacs, relacionados com as palavras-chave: "alojamento" e "conjunto". A caracterização foi feita a partir dos seguintes critérios: década da publicação, área de atuação dos autores, local da pesquisa e metodologia utilizada. Para realizar a análise qualitativa foram elaboradas as seguintes questões norteadoras: quais as possibilidades e limites existentes no SAC? Qual a atuação da equipe multiprofissional no Alojamento Conjunto?

A partir destas questões, identificamos as ideias centrais, as quais respondiam às questões acima, e dividimos temáticas em três grandes temas: o SAC como um espaço de promoção do Aleitamento Materno, de Vínculos e das Ações da Equipe Multiprofissional. 


\section{RESULTADOS, ANÁLISE E DISCUSSÃO}

\section{Caracterização dos artigos}

Há um desequilíbrio entre o volume de artigos publicados nas décadas de 1990 e 2000, sendo 61\% e $39 \%$, respectivamente. Acredita-se que tal resultado é justificado pelo fato da implantação do SAC ter ocorrido na década de 90 , o que instigou uma quantidade maior de docentes e discentes a pesquisar sobre o assunto. Na região sudeste houve predomínio das pesquisas realizadas (82\%), seguida da região sul (15\%) e nordeste (3\%). Não foram encontradas publicações sobre o tema proveniente das regiões norte e centro-oeste.

A maior parte dos autores é da área de Enfermagem (58\%), demonstrando maior interesse destes profissionais no SAC, considerando a participação direta dessa equipe no sistema. Em seguida, apresentam-se os autores atuantes na Medicina (21\%) e Nutrição (3\%), além de trabalhos realizados por equipes multiprofissionais formadas por enfermeiros, médicos, psicólogos, terapeutas ocupacionais e nutricionistas, totalizando 3\%.

\section{O Alojamento Conjunto como um espaço de promoção do Aleitamento Materno}

A implantação do Alojamento Conjunto, assim como a criação dos bancos de leite humano e de grupos de apoio à amamentação, imprimiram grandes mudanças nas políticas fundamentais na assistência ao binômio mãe-filho. Entretanto, ainda persistiam os índices elevados de desmame precoce ${ }^{(5)}$.

Considerando este fato, a OMS e o UNICEF, em 1992, idealizaram a Iniciativa Hospital Amigo da Criança, sendo esta incorporada pelo Ministério da Saúde como ação prioritária para promover, proteger e apoiar a amamentação. A iniciativa foi baseada nos "Dez Passos para o Sucesso da Amamentação", os quais tinham o objetivo de promover mudanças nos procedimentos hospitalares, em benefício do aleitamento natural ${ }^{(6)}$.

O Alojamento Conjunto proporciona contato constante entre o mãe e filho, sendo considerado um passo importante para o êxito da amamentação; possibilita a amamentação sob livre demanda (passo 8), presume que o recém-nascido não receba outro alimento senão o leite materno (passo 6) e que as mães participem dos cuidados de seus filhos, sob orientação da equipe de saúde. O Alojamento Conjunto tem como principal vantagem o estímulo ao aleitamento materno da forma mais natural possível, incentivando o vínculo mãe-filho e tendo a intenção de atuar como uma "escola de mães”, permitindo ministrar palestras sobre higiene e nutrição, além dos cuidados com o recém-nascido( ${ }^{(7)}$.

Entre as práticas realizadas no SAC relacionadas ao aleitamento materno, destacam-se: o favorecimento da precocidade da lactação devido à proximidade física do binômio e a flexibilidade dos horários; a promoção de incentivo, estímulo e motivação ao aleitamento materno, considerando que a amamentação precoce provoca a contração uterina e de seus vasos, atuando como profilaxia das hemorragias pósparto; a possibilidade de a mãe amamentar mais e por mais tempo, facilitando a apojadura e o estabelecimento da lactação. Considerando todos esses benefícios para a saúde do binômio, essas práticas, incentivadas desde a implantação do SAC, continuam vigentes na atualidade.

Porém, muitas práticas hospitalares são antiamamentação, como a separação do binômio e a alimentação suplementar antes da primeira mamada, esta ocorrendo tardiamente. No berçário, ainda existe a oferta de mamadeiras e bicos durante o período de internação, o que pode prejudicar o reflexo de sucção e o processo de lactação ${ }^{(8)}$. Vale ressaltar que o SAC deve propiciar ao binômio mãe-filho segurança, vínculo, cuidados adequados com o recém-nascido e educação em saúde ${ }^{(9)}$.

\section{O Alojamento Conjunto como um espaço de promoção de Vínculos}

As interações iniciais entre a mãe e o recémnascido são descritas como um processo comunicativo e de apego materno, considerando-se uma ação que estimula a adaptação mãe-filho, uma fase de ajustamento, na qual o bebê passa a fazer parte do mundo externo. Manter a mãe e a criança juntas logo após o nascimento estimula a operação de mecanismos sensoriais, hormonais, fisiológicos, imunológicos e comportamentais, que vinculam os pais ao bebê( $\hat{(}^{(10)}$. O aconchego resultante da interação sensorial estreita dá à criança a sensação de pertencimento, e referência insubstituível para a estruturação de sua personalidade. Há sistemas neuroquímicos, como os da ocitocina e vasopressina, desenvolvidos no cérebro da criança, que operam em sintonia com o afeto materno, reforçando o equilíbrio emocional ou gerando agressividade e outros comportamentos sociais. Seus 
efeitos dependem do vínculo afetivo que se estabelece entre a mãe, a criança, o pai e a família, como primeiro grupo social. Esses vínculos garantem relações estáveis ou podem ser a fonte da violência humana.

Dentro desta percepção, encontramos o SAC como um espaço citado por vários autores como sendo ideal para reforçar o relacionamento entre mãe, filho e também toda a família. Este relacionamento, ou vínculo, se inicia no momento da concepção, surge não só de uma complexa inter-relação de vários fatores de ordem genética, psicológica, mas também das vivências intrafamiliares e sociais, ou ainda observação e aprendizagem de valores culturais. Um período muito importante para o fortalecimento desse vínculo, e bastante estudado ultimamente, é o das primeiras horas e dias após o parto.

A separação do binômio no pós-parto imediato pode levar a mãe a um sentimento de competência diminuída e a manifestar menor afetividade. Tais comportamentos se devem a um estado hormonal diferenciado, logo após o parto, quando a mãe estaria mais propensa a receber o recém-nascido ${ }^{(11)}$.

O SAC favorece o relacionamento mãe-filho, proporciona satisfação, tranquilidade e confiança pessoal, a partir do momento em que as mães podem observar e atender seus filhos sempre que forem solicitadas. Elas passam a se sentir mais seguras e confiantes. Fora do Alojamento Conjunto, a mãe escuta o choro de criança e pensa que é o do seu filho e que nada está sendo feito para satisfazê-lo, enquanto que no Alojamento o choro é reduzido pelo pronto atendimento que a mãe oferece. Isso transmite segurança e diminui a ansiedade dos pais que permanecem com seus filhos, se comparados com aqueles que têm contato menor contato com a criança ${ }^{(12)}$.

A exclusão do pai do processo de parto e nascimento é muito presente, e ainda, não existe preparação dos homens para as tarefas mais complicadas de criar os filhos. Na prática profissional, pelo SAC, o pai está presente e interessado no bem-estar de sua mulher e filho, e é tratado como um membro qualquer da família( ${ }^{(12)}$.

De acordo com um estudo realizado em um hospital universitário de Porto Alegre, sobre a expectativa de alguns pais em relação aos cuidados prestados à mãe e ao recém-nascido no período pós-natal, foi manifestado o desejo dos homens de tomar conta desse binômio, adquirir experiência sobre o cuidado e, assim, trazer benefícios para todos. Porém, os pais também esperam encontrar dificuldades durante o período de hospitalização de sua família, como a não-participação do parto, preocupação com normas e rotinas inflexíveis, além da responsabilidade de cuidar dos filhos mais velhos $^{(12)}$.

Nesse espaço, a equipe multiprofissional necessita participar ativamente na inclusão do pai e de familiares no processo de formação de vínculo após o nascimento, através de contribuições educativas, que considerem a importância desse período, fundamental na vida do ser humano.

\section{O Alojamento Conjunto como um espaço de promoção das ações da equipe multiprofissional}

De acordo com o Ministério da Saúde, o SAC deve contar com uma equipe multiprofissional composta de um enfermeiro para cada 30 binômios, um auxiliar de enfermagem para cada 8 binômios, um obstetra para 20 mães, um pediatra para 20 recém-nascidos, além de assistente social, psicólogo e nutricionista ${ }^{(13)}$.

Essa equipe tem como objetivo realizar promoção da saúde e prevenção de eventuais problemas. O profissional deve ser capacitado para fornecer à mãe informações sobre o atual estado de saúde do binômio, avaliar suas condições físicas e emocionais, esclarecer sobre as rotinas gerais da unidade e sobre o autocuidado e cuidado ao recém-nascido. Cabe também à equipe oportunizar que a família, em especial o pai, participe de todo esse processo; acompanhar a evolução diária do binômio; reforçar orientações e detectar precocemente problemas, além de preparar a alta da mãe e do recém-nascido, revisando orientações dadas e fornecendo os encaminhamentos necessários. É possível, para o êxito do cuidado, que o profissional se mantenha atualizado quanto às ações educativas e que haja integração, entrosamento, interesse e cooperação mútua entre a equipe de saúde e a família, a fim de prover maior qualidade nas suas orientações.

Por meio da educação em saúde, pode-se observar uma oportunindade para o profissional educar de maneira significativa através de atividades, como os jogos educativos que buscam contribuir para ampliar o conhecimento das mães sobre a relevância do aleitamento materno, o autocuidado e os cuidados básicos ao recém-nascido; eles também proporcionam mais segurança e motivação, além de haver trocas de experiência e maior interação entre as participantes, não demandar custos elevados, possibilitar mudança da rotina hospitalar e revelar a motivação e interesse das mães ${ }^{(14)}$. 
O profissional deve valorizar os sentimentos das mães, usar linguagem clara e objetiva, mostrar disponibilidade e interesse em ouvir e esclarecer dúvidas e medos. Para isso, deve priorizar a promoção da saúde materna, através de informações sobre aleitamento materno, orientações sobre o cuidado com as mamas, prevenindo possíveis complicações como mastite e ingurgitamento ${ }^{(14)}$.

A comunicação verbal e a não-verbal são estratégias que devem ser utilizadas, através do silêncio, do toque, da paciência e da individualidade, mantendo os olhos no mesmo nível ou ficando face-a-face com a puérpera. Elas se constituem em ação terapêutica, uma forma de ouvir, dar atenção ou deixar chorar, principalmente no cuidado ao binômio mãe-filho e à família que passam por momentos de intensa angústia, tensão, medo e insegurança.

Em contrapartida, em alguns locais foi evidenciada escassez de recursos humanos, algumas equipes não trabalhavam em conjunto, levando a uma dicotomia dos cuidados. Outra lacuna constatada foi a falta de conscientização dos profissionais sobre a importância da educação em saúde no SAC.

\section{CONSIDERAÇÕES FINAIS}

Constatou-se, através da revisão bibliográfica, que o SAC apresenta inúmeras possibilidades. Uma delas, apontada por todos os autores, é a promoção e o incentivo ao aleitamento materno, que reduzem consequentemente a mortalidade neonatal. Elas possibilitam também, o conhecimento das necessidades do recémnascido, permitindo sua assistência integral. Outro aspecto são os benefícios para as mães, pois tais ações favorecem a aceitação da maternidade, proporcionando à puérpera um sentimento de bem-estar e praticidade, diminuindo assim sua ansiedade, e permitindo a troca de experiência entre as mães. $\mathrm{O}$ vínculo entre $\mathrm{o}$ trinômio mãe-filho-família, e deste com a equipe, também é reforçado no SAC, o qual diminui o abandono e a rejeição dos recém-nascidos e o risco de infecções cruzadas, além de permitir a educação em saúde.

Em contrapartida, percebeu-se, durante o estágio curricular, que poucos autores registraram limites, como a necessidade de maior área física nas maternidades, a redução de leitos, maior consumo de roupas e materiais, ocasionando dispêndio na assistência adequada ao binômio. Isso pode proporcionar angústia nas mães que não estão com seus filhos, acarretar perda de sono das mulheres e levá-las a crer que a equipe de enfermagem não está exercendo seu papel, transferindo-lhe a responsabilidade de todo o cuidado. Além disso, foi considerada a dificuldade de convivência entre pessoas com costumes e condições psicossociais diferentes, na mesma enfermaria.

Quanto à atuação da equipe multiprofissional, notou-se uma insuficiência de informação devido ao fato de haver pouco enfoque dos autores sobre o tema. Concluiu-se que os profissionais envolvidos com a proposta do SAC devem trabalhar com criatividade e atualização de conhecimento que estão diretamente ligados a ações educativas mais efetivas. A vivência de grupos educativos fortalece o cuidado e proporciona união de forças para combater os obstáculos, ocasionando a satisfação de toda a equipe e estando atentos e sensíveis às necessidades do binômio.

\section{REFERÊNCIAS}

1. Ungerer RLS, Miranda ATC. História do alojamento conjunto. J Pediatr. 1999;75(1):5-10.

2. Ministério da Saúde (BR). Portaria n. 1.016, de 26 de agosto de 1993: aprova as normas básicas para a implantação do sistema “Alojamento Conjunto”. Diário Oficial da União, Brasília, 1 set. 1993. Seção 1:13066.

3. Medeiros JB. Redação científica: a prática de fichamentos, resumos, resenhas. $6^{a}$ ed. São Paulo: Atlas; 2004.

4. Marconi MA, Lakatos EM. Metodologia do trabalho científico: procedimentos básicos, pesquisa bibliográfica, projeto e relatório, publicações e trabalhos científicos. $6^{a}$ ed. São Paulo: Atlas; 2001.

5. Vinagre RD, Diniz EMA, Vaz FAC. Leite humano: um pouco de sua história. Pediatria. 2001;23(4):340-5.

6. Ministério da Saúde (BR). Iniciativa Hospital Amigo da Criança IHAC [Internet]. Brasília: Ministério da Saúde;2008 [acesso em 20 mar 2008]. Disponível: http:/ /portal.saude.gov.br/portal/saude/cidadao/visualizar_ texto.cfm?idtxt=26348

7. Sakae PPO, Costa MTZ, Vaz FAC. Cuidados perinatais humanizados e o aleitamento materno promovendo a redução da mortalidade infantil. Pediatria. 2001;23(2):17987.

8. Pinto LM, Vitolo MR, Gírio LT, Léon MRAC, Zagari MCF, Farias NMF, et al. Aleitamento materno exclusivo em alojamento conjunto: avaliação da incidência e das

Cogitare Enferm. 2010 Abr/Jun; 15(2):334-9 
causas do uso de fórmulas. Rev Cienc Med PUCCAMP. 1996;5(2): 63-8.

9. Pinto LM, Vitolo MR, Sanches PO, Ferreira LBB. Caracterização e avaliação qualitativa do sistema de alojamento conjunto do Hospital e Maternidade Celso Pierro(HMCP)/PUC/Campinas. Pediatria. 1998;20(4):41621.

10. Vasconcelos SG, Paiva SS, Galvão MTG. Comunicação proxêmica entre mãe e filho em alojamento conjunto. Rev Enferm UERJ. 2006;14(1):37-42.

11. Figueiredo Júnior I. Alojamento conjunto: vantagens e desvantagens. J Bras Med. 1994;66(6):57-62.

12. Schmidt MLS, Bonilha ALL. Alojamento conjunto: expectativas do pai com relação aos cuidados de sua mulher e filho. Rev Gauch Enferm. 2003;24(3):316-24.

13. Ministério da Saúde (BR). Portaria n. 756, de 16 de dezembro de 2004: normas para o processo de habilitação do hospital amigo da criança integrado ao Sistema Único de Saúde - SUS. Diário Oficial da União, Brasília; 17 dez. 2004. Seção 1:99.

14. Fonseca LMN, Scochi CGS, Melo DF. Educação em saúde de puérperas em alojamento conjunto neonatal: aquisição de conhecimento mediado pelo uso de um jogo educativo. Rev Latino-Am Enfermagem. 2002;10(2): 166-71. 